\title{
CDH12 promotes the invasion of salivary adenoid cystic carcinoma
}

\author{
JIN-FENG WANG ${ }^{1 *}$, LIN SHE $^{1 *}$, BO-HUA SU ${ }^{1 *}$, LIN-CAN DING $^{1}$, \\ FEI-FEI ZHENG ${ }^{1}$, DA-LI ZHENG ${ }^{2}$ and YOU-GUANG LU ${ }^{1}$
}

\author{
${ }^{1}$ Department of Preventive Dentistry, Hospital of Stomatology, Fujian Medical University, \\ 246 Yangqiao Zhong Road, Fuzhou 350002; ${ }^{2}$ Department of Biochemistry and Molecular \\ Biology, Fujian Medical University, 88 Jiaotong Road, Fuzhou 350004, P.R. China
}

Received February 7, 2011; Accepted March 14, 2011

DOI: $10.3892 /$ or.2011.1286

\begin{abstract}
Cadherins are found in almost all living organisms. In addition to their role in the formation and maintenance of normal tissue architecture, cadherins seem to play a crucial role in the cell-cell interactions of cancer cells in tumorigenesis, invasion and metastasis. The aim of the present study was to identify the role of CDH12 in the invasion and metastasis of salivary adenoid cystic carcinoma (SACC). Real-time PCR results showed that CDH12 is abnormally expressed in the highly metastatic SACC cell line ACC-M, compared to ACC-2, a SACC cell line with low metastatic ability. CDH12 expression was significantly higher in clinical samples with metastasis and recurrence than in those without metastasis and recurrence $(\mathrm{P}<0.05)$, as demonstrated by immunohistochemical analysis. Overexpression of the $\mathrm{CDH} 12$ protein in ACC-M cells infected with an adenovirus vector containing CDH12 enhanced the invasive and migratory ability of ACC-M cells in vitro compared to ACC-M cells infected with empty vector. Likewise, knockdown of CDH12 by small interfering RNA efficiently inhibited the invasion and migration of ACC-M cells in vitro. These results indicate that $\mathrm{CDH} 12$ may play an important role in the invasion and metastasis of SACC.
\end{abstract}

\section{Introduction}

Salivary adenoid cystic carcinoma (SACC) is one of the most common malignancies of the salivary gland. Biological

Correspondence to: Dr You-Guang Lu, Department of Preventive Dentistry, Hospital of Stomatology, Fujian Medical University, 246 Yangqiao Zhong Road, Fuzhou 350002, P.R. China

E-mail: fjlyg63@163.com

Dr Da-Li Zheng, Department of Biochemistry and Molecular Biology, Fujian Medical University, 88 Jiaotong Road, Fuzhou 350004, P.R. China

E-mail: dalizheng@hotmail.com

*Contributed equally

Key words: salivary adenoid cystic carcinoma, CDH12, invasion properties of this carcinoma include aggressive growth, nerve and blood vessel invasion, distant metastases and high rates of recurrence, which result in poor patient survival. Surgical resection with adjuvant local radiation is the mainstay of treatment. Kokemueller et al reported that 74 patients with this disease had a relatively favorable 5-year overall survival rate $(71 \%)$ after treatment with surgical resection and local radiation at their department. However, their 10- and 15-year survival rates were poor (54 and 37\%, respectively) (1).

Cadherins are calcium-dependent cell-cell adhesion molecules that constitute a large superfamily that is involved in the formation and maintenance of histo-architecture (2). In addition, cadherins appear to play an important role in tumorigenesis, invasion and metastasis (3-5). Lu et al have generated gene expression profiles of SACC high and low metastasis cell lines using restriction fragment differential display-PCR (RFDD-PCR), which can screen related differentially expressed genes more accurately and efficiently (6). Using this method, we found that 14 genes of the Cadherin family are abnormally expressed in the highly metastatic SACC cell line ACC-M, indicating a potential role of cadherin family members in SACC invasion and metastasis.

The key members of the cadherin family include E-cadherin (encoded by CDH1), N-cadherin (encoded by $\mathrm{CDH} 2$ and CDH12), VE-cadherin (encoded by CDH5), R-cadherin (encoded by CDH4) and P-cadherin (encoded by CDH3), which exhibit different tissue distributions. Cadherins are the key transmembrane components of adherens junctions. They are composed of three segments: an extracellular domain responsible for cadherin-cadherin interactions; one transmembrane domain; and a highly conserved cytoplasmic domain that associates with actin filaments and serves to connect the outside of the cell to the cytoskeleton. In view of the important role of cadherins in the maintenance of normal tissue architecture, it is not surprising that cadherins are also involved in carcinoma progression. However, the effects of cadherin family members, especially CDH12, on SACC invasion and metastasis are currently poorly understood. In this study, we investigated the role of CDH12 in SACC invasion and metastasis. 
Table I. Ten types of cadherin genes and internal reference primers for real-time PCR.

\begin{tabular}{llll}
\hline Gene & Accession no. & \multicolumn{1}{c}{ Forward } & \multicolumn{1}{c}{ Reverse } \\
\hline ACTB & NM 001101 & CCTGGCACCCAGCACAAT & GGGCCGGACTCGTCATACT \\
CDH1 & NM004360.3 & GCTTCCCTCTTCATCTCCTGA & GCCACATTTCTTCTTGCTCCT \\
CDH2 & NM001792.3 & TAATGACAATGCCCCTCAAGTG & CAGCACAAGGATAAGCAGGATG \\
CDH3 & NM001793.4 & AGGAATGTGGGCAGTTTGACTT & ATAGCAACGCAACAGGGAAAAT \\
CDH8 & NM001796.2 & GGGCGAACTCTACTCTGTTGGT & TCTTTGTCTGTGGTGGTCAGGT \\
CDH9 & NM0016279.3 & CAGATTCGCTCAGTTCTTTGGA & TTTCCTTCCGAGATTGTTAGGC \\
CDH10 & NM006727.2 & AAATGTATGGTGGTGGGGAAAG & GGCTTGTTTGTGAGGTTGCTTA \\
CDH12 & NM004061.3 & ACCACAGCCACAGCAGACTTTA & GCCTGAGCACGAAGAGTGTAGA \\
CDH13 & NM001257.3 & AAGCAAGGTGGGTGGAAACTAA & GTGGATTCGGGACACATACAAA \\
CDH19 & NM021153.2 & TAATGAGTTGGGACCTCGCTTT & GGTTGTATTGATGCCTGTGAGC \\
CDH20 & NM031891.2 & CACGGATTCTAACCAGGAGGTC & ACAAAATGAGCAACACCAGCAC \\
\hline
\end{tabular}

\section{Materials and methods}

Cell line and tissues. The high and low metastasis cell lines (HLMCL) of SACC in our study was sponsored by the Oncology Laboratory in the Department of Stomatology in Shanghai 9th People's Hospital. Cells were grown in DMEM culture medium (HyClone, USA) supplemented with $10 \%$ fetal bovine serum (HyClone) in a $37^{\circ} \mathrm{C}$ incubator with humidified air containing $5 \% \mathrm{CO}_{2}$. Experiments were performed using cells in the exponential phase of growth. Tissue samples were obtained from the First Affiliated Hospital of the Fujian Medical University and the Fujian Medical University Union Hospital. Twenty-three cases with metastasis and recurrence and 57 cases without metastasis and recurrence were included. This study was approved by the Institutional Review Board of Fujian Medical University and the written informed consent was obtained from each participant.

Real-time PCR. Total RNA was extracted from $5 \times 10^{6}$ cells using TRIzol Reagent (Invitrogen, USA). RNA samples were diluted to the same concentration based on the absorbance at $260 \mathrm{~nm}$, then reverse transcribed to create cDNA using the PrimeScript ${ }^{\mathrm{TM}} \mathrm{RT}$ reagent kit (Takara, Japan). Real-time PCR was performed with SYBR-Green-PCR master mix (Takara). The reaction was carried out in a TP800 (Takara). The primers used for $\beta$-actin (ACTB) and for the 10 cadherin family genes are shown in Table I. The PCR was carried out as follows: initial denaturation at $95^{\circ} \mathrm{C}$ for $30 \mathrm{sec}$, followed by 40 cycles of $95^{\circ} \mathrm{C}$ for $10 \mathrm{sec}$ and $60^{\circ} \mathrm{C}$ for $30 \mathrm{sec}$ and then dissociation curve analysis. The fluorescence value of the first 12 cycles was set as baseline. The $\mathrm{Ct}$ values of all samples were adjusted. Data were analyzed using the method previously reported by Livak and Schmittgen (7).

Immunohistochemistry. For the immunohistochemical assays, $3-\mu \mathrm{m}$ thick tissue sections were mounted on slides coated with poly-L-lysine. After deparaffinization in xylene, these sections were rehydrated in a decreasing gradient of ethanol and washed for $10 \mathrm{~min}$ in phosphate-buffered saline (PBS) $(\mathrm{pH}$ 7.2). The endogenous peroxidase activity was inhibited by incubation in methanol containing $3 \% \mathrm{H}_{2} \mathrm{O}_{2}$ for $10 \mathrm{~min}$.
After several washes in PBS, the sections were blocked with a universal blocking reagent (Maxin, USA) for $10 \mathrm{~min}$ at room temperature. A rat monoclonal CDH12 (R\&D, USA) primary antibody was applied at a 1:200 $(10 \mu \mathrm{g} \mathrm{IgG} / \mathrm{ml})$ dilution for $1 \mathrm{~h}$ at room temperature. After several washes in PBS, the sections were incubated with a biotin-conjugated secondary antibody (Maxin) for $10 \mathrm{~min}$ at room temperature. After several washes in PBS, the sections were incubated with streptavidin-peroxidase (Maxin) for $10 \mathrm{~min}$ at room temperature. The sections were rinsed with PBS, and the antibody complexes were visualized by incubation with diaminobenzidine tetrahydrochloride (DAB) chromogen (Maxin). The sections were then counterstained with hematoxylin (Dako, Denmark), dehydrated, and examined by light microscopy. All slides were reviewed independently by two pathologists who were blinded to each other's readings. The staining results were assessed on a three-tier scale: negative was no staining, 1+ was weak staining and 2+ was strong staining. Immunohistochemical results were graded to 3 different scores (negative, positive and strong positive) as follows: negative was no staining or $1+$ staining in $\leq 30 \%$ of cells, positive was $1+$ staining in $>30 \%$ of cells or $2+$ staining in $<50 \%$ of cells and strong positive was $2+$ staining in $>50 \%$ of cells.

Construction of CDH12 adenoviral vector and infection. The CDH12 recombinant adenoviral expression vector (Ad-CDH12) was constructed using AdEasy XL Adenoviral Vector System (Stratagene, USA). Total RNA was extracted from ACC-M cells using TRIzol (Invitrogen), and cDNA was synthesized by reverse transcription using the PrimeScript RT reagent kit (Takara). NheI and XhoI sites were added to either side of the CDH12 gene, and the construct was amplified by nested PCR with PrimeSTAR HS DNA Polymerase (Takara), according to the supplier's protocol, using the primers indicated in Table II. After the cloning of CDH12 into the pMD18-T Vector (Takara) and sequencing analysis, the correct full-length coding sequence of CDH12 was subcloned into the shuttle vector pShuttle-IRES-hrGFP-1. The plasmids were used to transform competent BJ5183, containing the adenoviral backbone plasmid pAdEasy-1, to produce a recombinant adenovirus carrying the gene of interest. Then, 
Table II. Nested-PCR primers.

\begin{tabular}{lll}
\hline $\mathrm{CDH} 12$ & & \multicolumn{1}{c}{ Sequence of primers } \\
\hline Outer-primer & Forward & 5'-ACGGTTGATTTGACGGATTTCT-3' \\
& Reverse & 5'-CTTGTCCCAGAGTGTGTGTGTG-3' \\
Inter-primer & Forward & 5'-CTAGCTAGCATGCTTACAAGGAACTGTTT-3' \\
& Reverse & 5'-CCG $\underline{C T C G A G A G T G A C T T T A T C A G G G T T A T-3 '}$ \\
\hline
\end{tabular}

The forward inter-primer contains NheI restriction site (underlined and italic bases), and the reverse inter-primer contains XhoI restriction site (underlined and italic bases).

the recombinant adenovirus plasmids were produced in bulk using the XL10-Gold strain. The recombinant adenoviral construct was linearized with PacI and transfected into the packaging cell line, AD-293. After production, the adenovirus was amplified for three rounds. Adenovirus-GFP (Ad-GFP) was constructed and used as the control. ACC-M cells were infected with the adenoviral vector at the same multiplicity of infection (MOI).

RNA interference using small interfering RNA. Four siRNAs against CDH12 were designed and chemically synthesized (Shanghai GenePharma Co., Shanghai, China) for targeting different coding regions of $\mathrm{CDH} 12$. The sequence information is as follows: siRNA-1435 (5'-CCUAGAUAGAGAAGAG AAATT-3' and 5'-UUUCUCUUCUCUAUCUAGGTT-3'), siRNA-2371 (5'-GGACAGCUACUUUACAAUATT-3' and 5'-UAUUGUAAAGUAGCUGUCCTT-3'), siRNA-2932 (5'-GGCGUUGAUUGCAAUUCUATT-3' and 5'-UAGAAUU GCAAUCAACGCCTT-3') and siRNA-3434 (5'-GGCGAAG AAGAGAGUUAUATT-3' and 5'-UAUAACUCUCUUCU UCGCCTT-3'). In addition, a negative control, siRNA-NC (5'-UUCUCCGAACGUGUCACGUTT-3' and 5'-ACGUGA CACGUUCGGAGAATT-3'), was also synthesized. Each of the above siRNAs were individually transfected into ACC-M cells using Lipofectamine 2000 Transfection Reagent (Invitrogen), according to the manufacturer's instructions.

SDS-PAGE and Western blot analysis. Fifteen micrograms of total protein from cells lysis were separated by SDS-PAGE on $10 \%$ gels and electrophoretically transferred to PVDF membranes (Amersham Biosciences). The resulting membranes were blocked in $1 \%$ bovine serum albumin and incubated with primary antibodies against CDH12 (1:5000, Abcam, UK) or $\beta$-tubulin (1:1000, Santa Cruz Biotechnology, USA). After the membranes were washed with TBST, alkaline phosphataseconjugated secondary antibodies were added to recognize the primary antibodies, and the immunoreactive protein bands were visualized using CDP-Star reagents (Roche, IN, USA).

In vitro cell invasion assay. Cell invasion assays were performed using 24-well transwell chambers $(8 \mu \mathrm{m}$ pore size, BD Sciences, USA) coated with Matrigel (354480, BD Sciences). Cells were serum-starved overnight, trypsinized, and collected in DMEM containing 1\% FBS. A total of $1 \times 10^{5}$ cells were then suspended in $500 \mu \mathrm{l}$ of DMEM containing $1 \%$ FBS and added to the upper chamber, while $700 \mu \mathrm{l}$ of DMEM containing $10 \%$ FBS and $10-\mu \mathrm{g} / \mathrm{ml}$ fibronectin (356008, BD Sciences) were placed in the lower chamber. DMEM containing 1\% FBS was added to the lower chamber in control wells. After $48 \mathrm{~h}$ of incubation, Matrigel and cells remaining in the upper chamber were removed with cotton swabs. Cells on the lower surface of the membrane were stained with crystal violet. Cells in at least five random microscopic fields (at x200) were counted and photographed. All experiments were repeated three times.

In vitro cell migration assays. Cell migration was evaluated using transwell assay and the scratch wound assay. The migration assay using transwell inserts $(8 \mu \mathrm{m}$ pore size, BD Sciences) was performed to assess cell motility using a method similar to that used in the transwell invasion chamber, except that transwell chamber was not coated with Matrigel. The wound-healing assay was performed as follows. Each group of ACC-M cells was seeded into wells of 6-well culture plates at the proper density to form monolayers. A straight scratch was made using a $10-\mu 1$ pipette tip on the bottom of the culture plate. After washing with serum-free medium, the relative width of the scratch wounds were measured and recorded under an inverted microscope. Next, the cells were grown in DMEM culture medium with $0.1 \%$ fetal bovine serum (FBS) for $48 \mathrm{~h}$, and the migration of the cultured cells was observed and photographed. The experiments were repeated three times.

Statistical analysis. Data are expressed as means \pm SD. The statistical analysis of in vitro cell invasion and migration assays was performed using Student's t-test. Differences were considered to be statistically significant at $\mathrm{P}<0.05$.

\section{Results}

Cadherins are abnormally expressed in the highly metastatic cell line ACC-M. Cadherin family members play an important role in the maintenance of normal tissue architecture and are implicated as modulators of development processes by controlling cell polarity, cell sorting and cell movements. In a previous study, we found that 14 cadherin genes were abnormally expressed based on gene expression profiles of the adenoid cystic carcinoma cell lines, ACC-M and ACC-2 (6). In this study, real-time PCR was first used to verify the differential expression of the 10 cadherin genes in ACC-M and ACC- 2 cells. The results showed that 9 out of 10 cadherin 


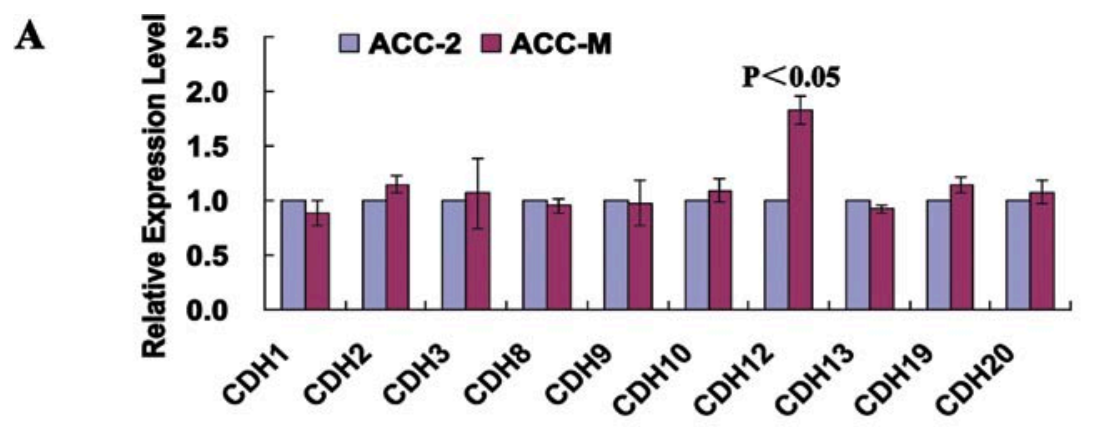

B
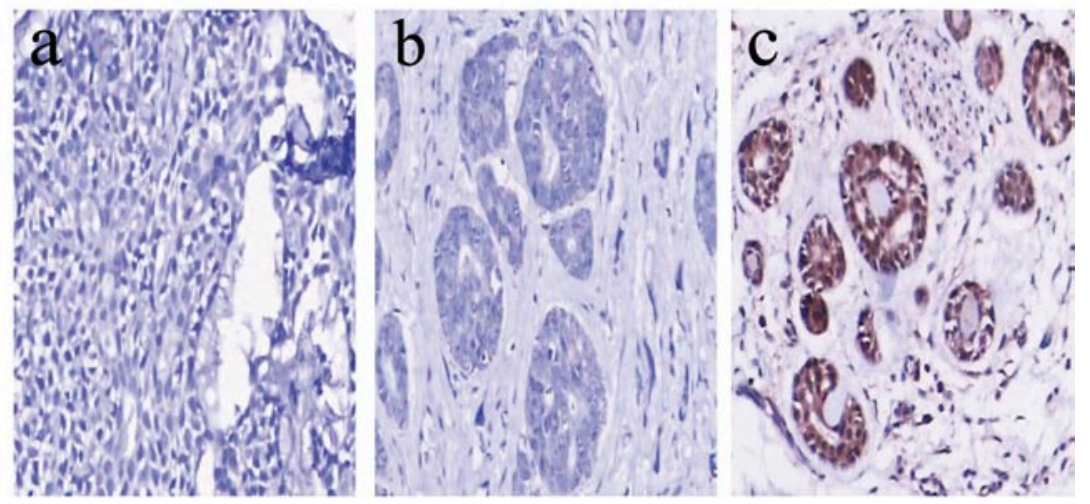

Figure 1. The expression analysis of CDH12 levels in salivary adenoid cystic carcinoma by real-time PCR and immunohistochemistry (DAB x400). (A) The comparison of the relative signal strength between the individual genes of the cadherin family and internal reference primer in the two cell lines by real-time PCR; (B) (a) The lack of expression of CDH12 in SACC tissues without metastasis and recurrence; (b) Positive staining for CDH12 in SACC cancer cells; (c) Strong immunopositivity for CDH12 in SACC cancer cells.

Table III. The expression of CDH12 in tissues of adenoid cystic carcinoma with or without metastasis and recurrence cases.

\begin{tabular}{lccccc}
\hline Group & Cases & Negative & Positive & Strong positive & P-value $^{\text {a }}$ \\
\hline Metastasis or recurrence & 23 & 3 & 6 & 15 & 0.024 \\
Non-metastasis & 57 & 5 & 33 & 19 & \\
\hline
\end{tabular}

${ }^{\mathrm{a}}$ Rank-sum test $\mathrm{Z}=-2.260$.

genes showed no difference in expression between these 2 cell lines, whereas CDH12 was up-regulated in the highly metastatic adenoid cystic carcinoma cell line, ACC-M, compared to the low metastatic cell line, ACC-2 (Fig. $1 \mathrm{~A}, \mathrm{P}<0.05, \mathrm{n}=3$ ). $\mathrm{CDH} 4$ and CDH5 were also abnormally expressed in the ACC-M cell line (unpublished data).

CDH12 is overexpressed in clinical metastatic tissue samples. To further explore the role of $\mathrm{CDH} 12$ in the metastasis and progression of adenoid cystic carcinoma, CDH12 expression in 80 tissue samples of adenoid cystic carcinoma was examined by immunohistochemistry. Of these samples, 23 cases had metastasis and recurrence; the other 57 cases were without metastasis and recurrence and served as controls. As shown in Table III and Fig. 1B, the expression levels of CDH12 were significantly higher in the tissues with metastasis and recurrence compared to those of control samples $(\mathrm{P}<0.05)$. This result indicates that $\mathrm{CDH} 12$ may play an important role in the metastasis of adenoid cystic carcinoma.
Overexpression of $\mathrm{CDH} 12$ promotes the invasiveness and motility of ACC-M cells. To further examine whether CDH12 affects the invasiveness of salivary adenoid cystic carcinoma, ACC-M cells were infected with the recombinant adenovirus (Ad-CDH12) carrying the correct full-length coding sequence of $\mathrm{CDH} 12$, and cell invasion and migration assays were performed to assess cell motility. After infection with Ad-CDH12, the expression of CDH12 protein in ACC-M cells was up-regulated compared to those cells infection with the control adenovirus (Ad-GFP) (Fig. 2A). As shown in Fig. 2B and C, the invasive ability of ACC-M cells infected with Ad-CDH12 was significantly enhanced compared to cells infected with Ad-GFP. The overexpression of CDH12 also enhanced the migration of ACC-M cells, both in the Transwell assay (Fig. 3A and B, $\mathrm{P}<0.05, \mathrm{n}=3$ ) and the wound-healing assay (Fig. 3C). These results suggest that the overexpression of $\mathrm{CDH} 12$ may promote the invasion and migration of ACC-M cells.

Knockdown of CDH12 inhibits the invasiveness and motility of ACC-M cells. To further verify the contribution of CDH12 
$\mathbf{A}$

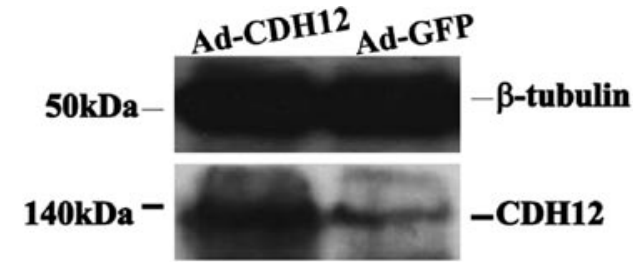

B

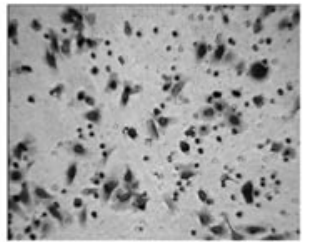

Ad-GFP

C

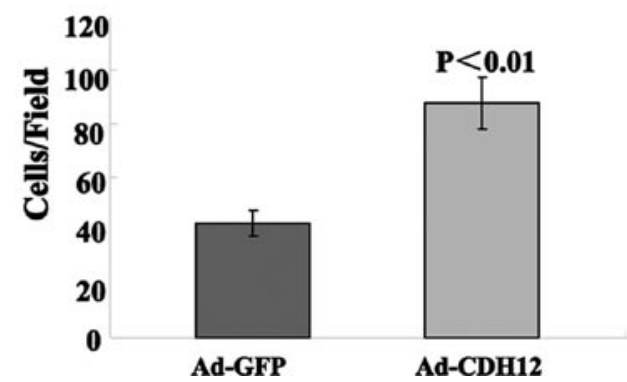

Figure 2. The overexpression of CDH12 facilitates the invasion of ACC-M cells. (A) Western blot analysis of the overexpression of CDH12 in ACC-M cells infected with adenoviral vector; (B) Each panel represents the results of cell invasion assays using ACC-M cells; (C) Cells that invaded the Matrigel layer were stained, photographed and measured by counting the invaded cells. The counts of the invaded cells are presented as mean values per field from at least five randomly selected low-powered fields (x200) from three independent experiments (error bars, mean $\pm \mathrm{SD}$ ).

to the invasion of salivary adenoid cystic carcinoma, we next employed small interfering RNA (siRNA)-mediated knockdown of CDH12 in ACC-M cells and performed cell invasion and migration assays. siRNAs targeting CDH12 (siRNA-1435, siRNA-2371, siRNA-2932 and siRNA-3434) efficiently reduced $\mathrm{CDH} 12$ expression in ACC-M cells compared to the negative control, siRNA-NC, as demonstrated by Western blot analysis (Fig. 4A). The two most effective siRNAs, siRNA-1435 and siRNA-3434, were selected for use in further experiments. As shown in Fig. 4B and C, the down-regulated expression of $\mathrm{CDH} 12$ significantly inhibited the invasion $(\mathrm{P}<0.05, \mathrm{n}=3)$ and migration of ACC-M cells (Fig. 5, $\mathrm{P}<0.05$, $\mathrm{n}=3$ ). These results confirm that $\mathrm{CDH} 12$ may contribute to the migration and invasion of adenoid cystic carcinoma cells.

\section{Discussion}

Invasion and metastasis are essential for tumor progression and are important indicators of malignancy and major causes of mortality in SACC. Although the 5-year survival of SACC patients treated with surgical resection and local radiation is deceptively high (71\%), the long-term survival is poorer, with only $37 \%$ of patients alive at 15 years (1). Thus, defining the mechanisms regulating SACC invasion and metastasis is important for not only explaining the pathogenesis of SACC, but also for prevention and treatment of SACC to improve the overall survival of SACC patients.

Cadherins constitute a large family of calcium-dependent transmembrane glycoproteins with several unique members. The classical cadherins comprise a subgroup that includes E-cadherin, N-cadherin, VE-cadherin and P-cadherin. Through their homophilic binding interactions, they play crucial roles in maintaining normal tissue architecture and conferring cellular adhesion specificities (8-10). Moreover, cadherins have been implicated as modulators of cell polarity, cell sorting, cell movements and tissue morphogenesis in development (11). Cadherins have also been demonstrated to play important roles in the cell-cell interactions of cancer cells during tumorigenesis, invasion and metastasis (12-17). Among the cadherins,
$\mathbf{A}$

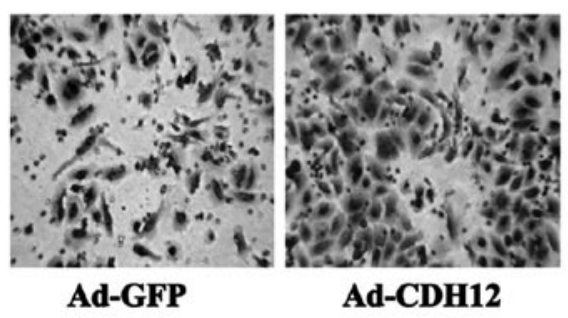

B

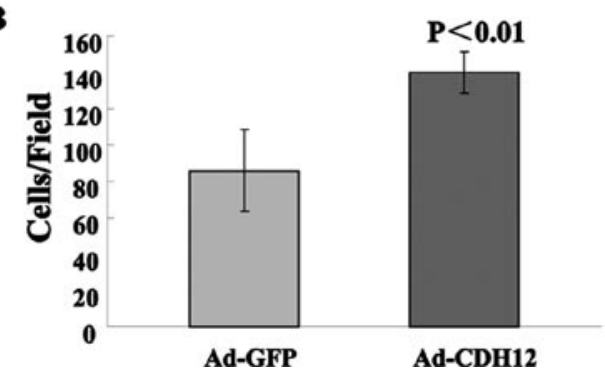

C

$\mathbf{0 ~ h}$
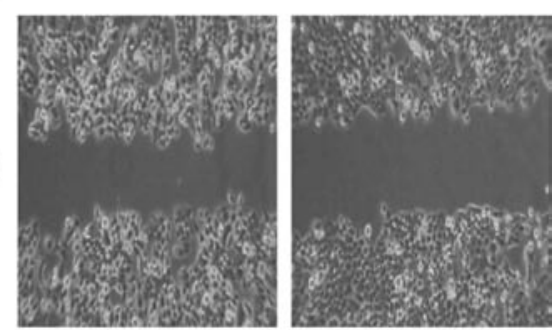

$48 \mathrm{~h}$

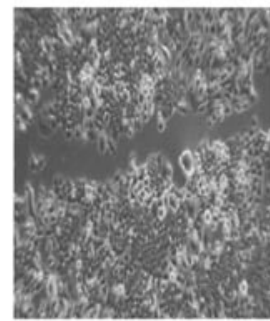

Ad-GFP

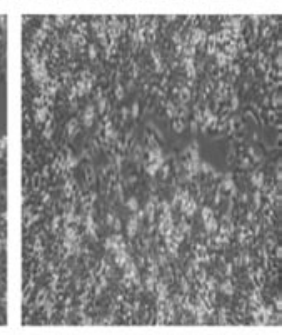

Ad-CDH12

Figure 3. The overexpression of CDH12 facilitates the migration of ACC-M cells. (A) Each panel represents the results of cell migration assays using ACC-M cells; (B) The number of cells migrated through uncoated filters (i.e., no Matrigel), which represents the migratory ability of ACC-M cells; (C) A photomicrograph of scratch wounds made in the infected ACC-M cell layer shows enhanced cellular motility in the adenovirus Ad-CDH12 infected cells compared with the control adenovirus Ad-GFP transfectants $48 \mathrm{~h}$ after cell seeding. 


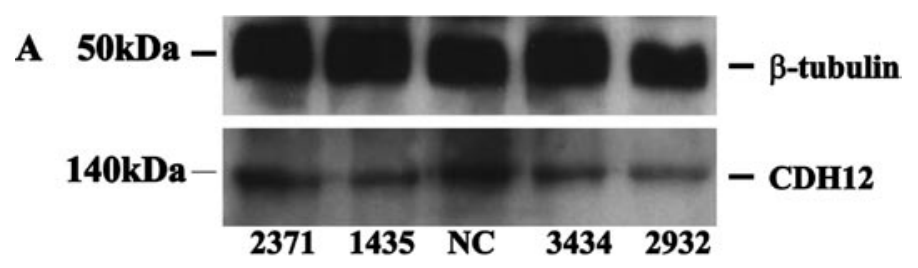

B

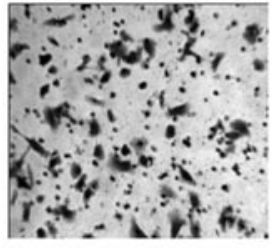

siRNA-NC

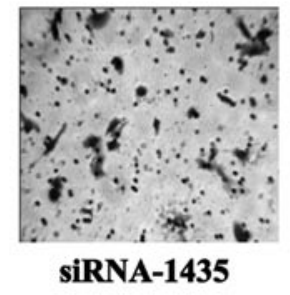

SiRNA-1435

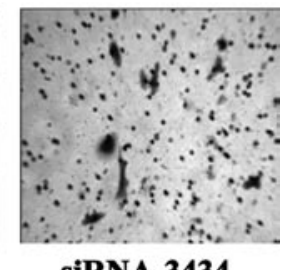

SiRNA-3434

C

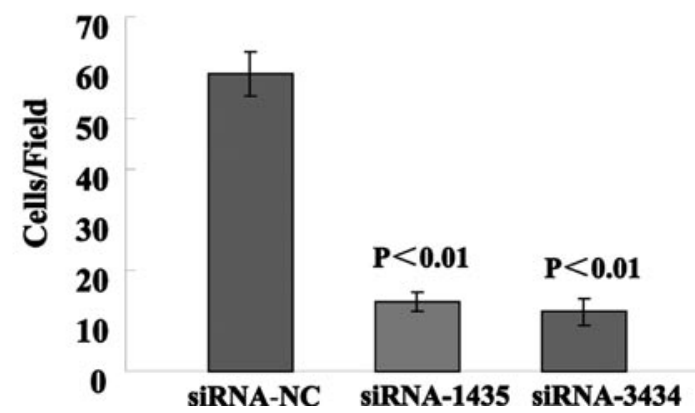

Figure 4. The knockdown of CDH12 inhibits the invasion of ACC-M cells. (A) Western blot analysis of knockdown of CDH12 expression in ACC-M cells with siRNAs; (B) Each panel represents the results of cell invasion assays using ACC-M cells; (C) Cells that invaded the Matrigel layer were stained. The counts of the invaded cells are presented as mean values per field from at least five randomly selected low-powered fields (x200) from three independent experiments (error bars, mean $\pm \mathrm{SD}$ ).

A

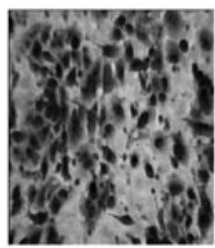

SiRNA-NC

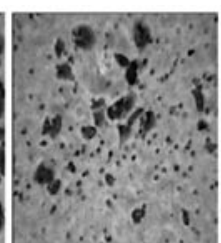

siRNA-1435

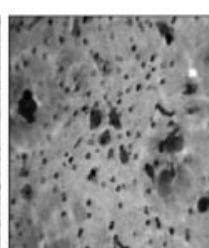

siRNA-3434

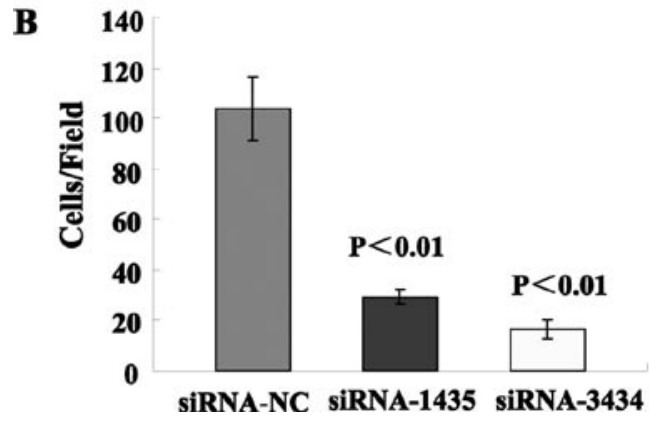

C

$\mathbf{0 ~ h}$
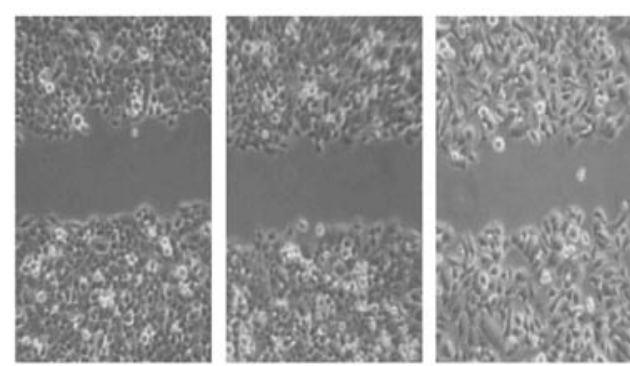

$48 \mathrm{~h}$

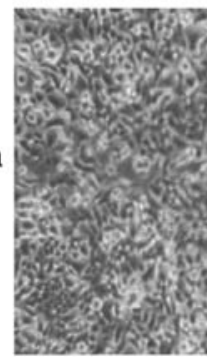

SiRNA-NC

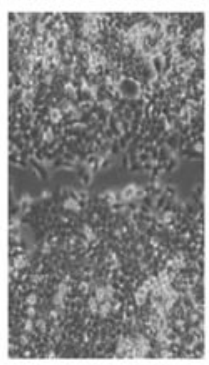

siRNA-1435

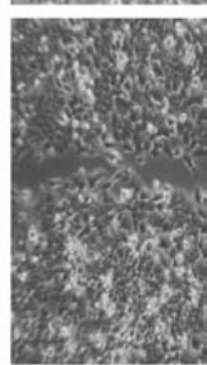

siRNA-3434

Figure 5. The knockdown of CDH12 inhibits the migration of ACC-M cells. (A) Each panel represents the results of cell migration assays using ACC-M cells; (B) The number of cells migrated through uncoated filters (i.e., without Matrigel), which represents the migratory ability of ACC-M cells; (C) A photomicrograph of scratch wounds made in the transfected ACC-M cell layer shows reduced cellular motility in the CDH12 siRNA transfection group compared to the negative control siRNA group $48 \mathrm{~h}$ after cell seeding.

the most focus has been drawn on E-cadherin. Numerous studies have shown that disruption of the E-cadherin/catenin complex in epithelial cells results in biologically aggressive tumor cells, implicating E-cadherin as the product of a tumor suppressor gene (18). Research carried out by Zhang et al found that E-cadherin/catenin complex expression was down- 
regulated in adenoid cystic carcinoma of the salivary glands, and the expression levels of E-cadherin/catenin complex were positively correlated to the differentiation of ACC tumor cells (19). Furthermore, Lai et al reported that E-cadherin expression was correlated with nerve invasion, regional recurrence and distant metastasis (20).

Another member of the cadherin family, N-cadherin (encoded by $\mathrm{CDH} 12$ ), has also gained increasing attention. According to the literature, $\mathrm{N}$-cadherin is aberrantly expressed in many human carcinomas, including breast cancer, thyroid, bladder and prostate carcinomas (21). To assess the expression pattern of cadherin family members in SACC, we examined the expression of 10 cadherin genes by real-time PCR in HLMCL of SACC. The results showed that expression of CDH12 was up-regulated in the highly metastatic SACC cell line, ACC-M, compared to expression in the low metastatic SACC cell line, ACC-2. Immunohistochemical analyses demonstrated that the expression of CDH12 was significantly higher in tissue samples from patients with metastasis than without. These data indicate a potential role for $\mathrm{CDH} 12$ in SACC invasion and metastasis. To further verify the role of CDH12 in SACC, CDH12 was overexpressed relative to normal levels in ACC-M cells using an adenovirus expression system. Along with increased $\mathrm{CDH} 12$ levels, invasion and migration of ACC-M cells were significantly enhanced in our study. The converse results were obtained when knockdown of CDH12 with siRNA decreased invasion and migration, which further confirmed the role of CDH12 in the invasion and migration of SACC.

Previous studies have shown that the high levels of $\mathrm{N}$-cadherin expression in the most invasive carcinoma cells were inversely correlated with the levels of E-cadherin expression, suggesting the possible role of $\mathrm{N}$-cadherin in facilitating invasion and metastasis $(18,22-24)$. Efforts have been made to show that $\mathrm{N}$-cadherin contributed to the progression of squamous cell carcinoma (SCC) of the tongue and was tightly associated with lymph node metastasis. Substantially decreased invasion was also observed in the tongue SCC cell line, Tca8113, after transfection with N-cadherin siRNAs, compared to that of control siRNA (25). Similar findings were reported in esophageal squamous cell carcinoma (ESCC). Moreover, the expressions of MMP-9 was also down-regulated in ESCC cell line EC9706 after transfection with N-cadherin shRNA (26). Additionally, the knockdown of N-cadherin in EC9706 could arrest cell cycle at G0/G1 phase, induce cell apoptosis, and inhibit tumor formation in vivo (27). It is known that growth factor receptors regulate many aspects of cell behavior, including cell motility and invasion (28). A number of studies have demonstrated that $\mathrm{N}$-cadherin facilitates dimerization of the fibroblast growth factor (FGF) receptor to initiate growth factor-independent signaling and mediate ligand-dependent FGF receptor signaling (29-31). For example, a small protein called NHERF acts as a scaffold to link $\mathrm{N}$-cadherin and $\beta$-catenin to the platelet-derived growth factor receptor (PDFR), and this complex of proteins is localized to the leading edge of migrating tumor cells, where it promotes motility (32). Studies have also suggested that N-cadherin might interact with other growth factor receptors on tumor cells, such as TGF $\beta 1$ (33). In addition, Guo et al reported that branched $\mathrm{N}$-glycan expression at three of the eight putative
$\mathrm{N}$-glycosylation sites in the EC2 and EC3 domains of the $\mathrm{N}$-cadherin sequence regulated $\mathrm{N}$-cadherin-mediated cell-cell contact formation, outside-in signaling, and cell migration, and this glycosylation is likely to significantly contribute to the increase in the migratory/invasive phenotype of cancer cells that results when GnT-V activity is up-regulated by oncogene signaling (34).

Invasion and metastasis are the most important biological markers of malignant tumors, and represent complex, continuous and multi-staged processes, in which both known and unknown genes are involved. In gene therapy, which is a very promising treatment for cancer, an important question is to determine which genes might be the best gene therapy targets. Therefore, it is important to define the crucial genes involved in cancer.

In our study, we demonstrated that $\mathrm{CDH} 12$ could promote the invasion of salivary adenoid cystic carcinoma. Based on this information, we concluded that $\mathrm{CDH} 12$ is of vital importance in the invasion and metastasis of SACC, although it may not be the only critical factor. Therefore, the results suggest the potential role of $\mathrm{CDH} 12$ as a candidate target in cancer therapy. Further investigations and elucidations are warranted to more precisely determine the roles of CDH12 in SACC.

\section{Acknowledgements}

This study was supported by the Key Project of Science and Technology Foundation of Fujian Province of China (No.2008Y0041) and the Natural Science Foundation of Fujian Province of China (No.C0710011).

\section{References}

1. Kokemueller H, Eckardt A, Brachvogel P and Hausamen JE: Adenoid cystic carcinoma of the head and neck - a 20 years experience. Int J Oral Max Surg 33: 25-31, 2004.

2. Takeichi M: The cadherins: cell-cell adehesion molecules controlling animal morphogenesis. Development 102: 639-655, 1988.

3. Cheung LW, Leung PC and Wong AS: Cadherin switching and activation of p120 catenin signaling are mediators of gonadotropin-releasing hormone to promote tumor cell migration and invasion in ovarian cancer. Oncogene 29: 2427-2440, 2010.

4. Herve W, Ying C, Zev AW, et al: Phospho-Akt pathway activation and inhibition depends on N-cadherin or phospho-EGFR expression in invasive human bladder cancer cell lines. Urol Oncol 28: 180-188, 2010.

5. Fougner SL, Lekva T, Borota OC, Hald JK, Bollerslev J and Berg JP: The expression of E-cadherin in somatotroph pituitary adenomas is related to tumor size, invasiveness, and somatostatin analog response. J Clin Endocrinol Metab 95: 2334-2342, 2010.

6. Ding LC, She L, Zheng DL, et al: Notch-4 contributes to the metastasis of salivary adenoid cystic carcinoma. Oncol Rep 24: 363-368, 2010.

7. Livak KJ and Schmittgen TD: Analysis of relative gene expression data using real-time quantitative PCR and the 2(-Delta Delta C(T)) method. Methods 25: 402-408, 2001.

8. Takeichi M: Cadherins: a molecular family important in selective cell-cell adhesion. Annu Rev Biochem 59: 237-252, 1990.

9. Takeichi M: Cadherin cell adhesion receptors as a morphogenetic regulator. Science 251: 1451-1455, 1991

10. Miyatani S, Shimamura K, Hatta M, et al: Neural cadherin: role in selective cell-cell adhesion. Science 245: 631-635, 1989.

11. Halbleib JM and Nelson WJ: Cadherins in development: cell adhesion, sorting, and tissue morphogenesis. Genes Dev 20: 3199-3214, 2006.

12. Behrens J, Mareel MM, van Roy FM and Birchmeier W: Dissecting tumor cell invasion: epithelial cells acquire invasive properties after the loss of uvomorulin-mediated cell-cell adhesion. J Cell Biol 108: 2435-2447, 1989. 
13. Chen WC and Obrink B: Cell-cell contacts mediated by E-cadherin (uvomorulin) restrict invasive behavior of L-cells. J Cell Biol 114: 319-327, 1991.

14. Navarro P, Gomez M, Pizarro A, Gamallo C, Quintanilla M and Cano A: A role for the E-cadherin cell-cell adhesion molecule during tumor progression of mouse epidermal carcinogenesis. J Cell Biol 115: 517-533, 1991.

15. Vleminckx K, Vakaet L Jr, Mareel M, Fiers W and van Roy F: Genetic manipulation of E-cadherin expression by epithelial tumor cells reveals an invasion suppressor role. Cell 66: 107-119, 1991.

16. Hamaguchi M, Matsuyoshi N, Ohnishi Y, Gotoh B, Takeichi M and Nagai Y: p60v-src causes tyrosine phosphorylation and inactivation of the $\mathrm{N}$-cadherin-catenin cell adhesion system. EMBO J 12: 307-314, 1993.

17. Kawamura-Kodama K, Tsutsui J, Suzuki ST, Kanzaki T and Ozawa M: N-cadherin expressed on malignant T cell lymphoma cells is functional, and promotes heterotypic adhesion between the lymphoma cells and mesenchymal cells expressing N-cadherin. J Invest Dermatol 112: 62-66, 1999.

18. Islam S, Carey TE, Wolf GT, Wheelock MJ and Johnson KR Expression of $\mathrm{N}$-cadherin by human squamous carcinoma cells induces a scattered fibroblastic phenotype with disrupted cell-cell adhesion. J Cell Biol 135: 1643-1654, 1996.

19. Zhang ZY, Wu YQ, Zhang WG, Tian Z and Cao J: The expression of E-cadherin-catenin complex in adenoid cystic carcinoma of salivary glands. Chin J Dent Res 3: 36-39, 2000.

20. Lai FY, Zhang Q, Wu QL, Qing J and Cao Y: Expression and significance of E-cadherin in adenoid cystic carcinoma of the salivary glands. Chin J Cancer 26: 1025-1028, 2007.

21. Gloushankova NA: Changes in regulation of cell-cell adhesion during tumor transformation. Biochem J 73: 742-750, 2008.

22. Hazan RB, Kang L, Whooley BP and Borgen PI: N-cadherin promotes adhesion between invasive breast cancer cell lines and the stroma. Cell Adhes Commun 4: 399-411, 1997.

23. Li Z, Gallin WJ, Lauzon G and Pasdar M: L-CAM expression induces fibroblast-epidermoid transition in squamous carcinoma cells and down-regulates the endogenous N-cadherin. J Cell Sci 111: 1005-1019, 1998.
24. Chung SS, Mo MY, Silvestrini B, Lee WM and Cheng CY: Rat testicular N-cadherin: its complementary deoxyribonucleic acid cloning and regulation. Endocrinology 139: 1853-1862, 1998.

25. Li S, Jiao J, Lu Z and Zhang M: An essential role for $\mathrm{N}$-cadherin and $\beta$-catenin for progression in tongue squamous cell carcinoma and their effect on invasion and metastasis of Tca8113 tongue cancer cells. Oncol Rep 21: 1223-1233, 2009.

26. Li K, He W, Lin N, Wang X and Fan QX: N-cadherin knockdown decreases invasiveness of esophageal squamous cell carcinoma in vitro. World J Gastroenterol 15: 697-704, 2009.

27. Li K, He W, Lin N, Wang X and Fan QX: Down-regulation of N-cadherin expression inhibits invasiveness, arrests cell cycle and induces cell apoptosis in esophageal squamous cell carcinoma. Cancer Invest 28: 479-486, 2010.

28. McKay MM and Morrison DK: Integrating signals from RTKs to ERK/MAPK. Oncogene 26: 3113-3121, 2007.

29. Doherty P, Williams G and Williams EJ: CAMs and axonal growth: a critical evaluation of the role of calcium and the MAPK cascade. Mol Cell Neurosci 16: 283-295, 2000.

30. Skaper SD, Moore SE and Walsh FS: Cell signalling cascades regulating neuronal growth-promoting and inhibitory cues. Prog Neurobiol 65: 593-608, 2001.

31. Suyama K, Shapiro I, Guttman M and Hazan RB: A signaling pathway leading to metastasis is controlled by $\mathrm{N}$-cadherin and the FGF receptor. Cancer Cell 2: 301-314, 2002.

32. Theisen CS, Wahl JK, Johnson KR and Wheelock MJ: NHERF links the N-cadherin/catenin complex to the platelet-derived growth factor receptor to modulate the actin cytoskeleton and regulate cell motility. Mol Biol Cell 18: 1220-1232, 2007.

33. Diamond ME, Sun L, Ottaviano AJ, Joseph MJ and Munshi HG Differential growth factor regulation of $\mathrm{N}$-cadherin expression and motility in normal and malignant oral epithelium. J Cell Sci 121: 2197-2207, 2008

34. Guo HB, Johnson H, Randolph M and Pierce M: Regulation of homotypic cell-cell adhesion by branched $\mathrm{N}$-glycosylation of $\mathrm{N}$-cadherin extracellular EC2 and EC3 domains. J Biol Chem 284: 34986-34997, 2009. 